\title{
BMJ Open Effectiveness of pharmacist-led medication reconciliation programmes on clinical outcomes at hospital transitions: a systematic review and meta-analysis
}

\author{
Alemayehu B Mekonnen, ${ }^{1,2}$ Andrew J McLachlan,, ${ }^{1,3}$ Jo-anne E Brien ${ }^{1,4}$
}

To cite: Mekonnen $A B$, McLachlan AJ, Brien JE. Effectiveness of pharmacistled medication reconciliation programmes on clinical outcomes at hospital transitions: a systematic review and meta-analysis. BMJ Open 2016;6:e010003. doi:10.1136/bmjopen-2015010003

- Prepublication history and additional material is available. To view please visit the journal (http://dx.doi.org/ 10.1136/bmjopen-2015010003).

Received 17 September 2015 Revised 26 November 2015 Accepted 18 December 2015

CrossMark

For numbered affiliations see end of article.

Correspondence to Alemayehu B Mekonnen; aber5592@uni.sydney.edu.au

\section{ABSTRACT}

Objectives: Pharmacists play a role in providing medication reconciliation. However, data on effectiveness on patients' clinical outcomes appear inconclusive. Thus, the aim of this study was to systematically investigate the effect of pharmacist-led medication reconciliation programmes on clinical outcomes at hospital transitions.

Design: Systematic review and meta-analysis. Methods: We searched PubMed, MEDLINE, EMBASE, IPA, CINHAL and PsycINFO from inception to December 2014. Included studies were all published studies in English that compared the effectiveness of pharmacist-led medication reconciliation interventions to usual care, aimed at improving medication reconciliation programmes. Meta-analysis was carried out using a random effects model, and subgroup analysis was conducted to determine the sources of heterogeneity.

Results: 17 studies involving 21342 adult patients were included. Eight studies were randomised controlled trials (RCTs). Most studies targeted multiple transitions and compared comprehensive medication reconciliation programmes including telephone followup/home visit, patient counselling or both, during the first 30 days of follow-up. The pooled relative risks showed a more substantial reduction of $67 \%, 28 \%$ and $19 \%$ in adverse drug event-related hospital revisits (RR $0.33 ; 95 \% \mathrm{Cl} 0.20$ to 0.53 ), emergency department (ED) visits (RR $0.72 ; 95 \% \mathrm{Cl} 0.57$ to 0.92 ) and hospital readmissions ( $\mathrm{RR} 0.81 ; 95 \% \mathrm{Cl} 0.70$ to 0.95 ) in the intervention group than in the usual care group, respectively. The pooled data on mortality (RR 1.05; $95 \% \mathrm{Cl} 0.95$ to 1.16 ) and composite readmission and/ or ED visit (RR $0.95 ; 95 \% \mathrm{Cl} 0.90$ to 1.00 ) did not differ among the groups. There was significant heterogeneity in the results related to readmissions and ED visits, however. Subgroup analyses based on study design and outcome timing did not show statistically significant results.

Conclusion: Pharmacist-led medication reconciliation programmes are effective at improving post-hospital healthcare utilisation. This review supports the implementation of pharmacist-led medication

\section{Strengths and limitations of this study}

- This is the first systematic review investigating the effect of pharmacist-led medication reconciliation programmes on clinical outcomes.

- In some of the clinical outcomes evaluated, there is substantial statistical heterogeneity and we could not identify the source of variation among the studies.

- The inclusion of non-controlled studies might affect the quality of evidence as seen by the high risk of bias in these groups of studies.

reconciliation programmes that include some component aimed at improving medication safety.

\section{INTRODUCTION}

Medication reconciliation has been recognised as a major intervention tackling the burden of medication discrepancies and subsequent patient harm at care transitions. ${ }^{1}$ Unjustifiable medication discrepancies are responsible for more than half of the medication errors occurring at transitions in care, when patients move in and out of hospital or get transferred to the care of other healthcare professionals, ${ }^{2}$ and up to one-third could have the potential to cause harm. ${ }^{3}$ Incidence of unintentional medication changes is common at care transitions, ${ }^{3-8}$ and is one of the reasons for a huge utilisation of healthcare resources. ${ }^{9-13}$ Medication reconciliation as a medication safety strategy has been championed by a number of healthcare organisations. It was first adopted in 2005 as a National Patient Safety Goal (NPSG) by the Joint Commission ${ }^{14}$ and, later, the WHO and collaborators $^{15-17}$ involved themselves in endorsing this strategy across many countries. 
Despite these efforts, implementation of a medication reconciliation service is a hospital-wide challenge, ${ }^{18}$ and there is no previous clinical evidence as to which member of the healthcare profession(s) or which strategies effectively perform medication reconciliation. ${ }^{19} \mathrm{~A}$ number of medication reconciliation strategies have been utilised for safe patient transitions: use of electronic reconciliation tools, ${ }^{20-22}$ standardised forms ${ }^{23} 24$ and collaborative models, ${ }^{25}{ }^{26}$ as well as patient engagement ${ }^{27}$ and pharmacist-led approaches. ${ }^{28} 29$

The impact of medication reconciliation on clinical outcomes at hospital transitions has been reported, however, two recently published systematic reviews ${ }^{30} 31$ have ascertained that the benefit as a patient safety strategy is not clear. Both studies have inconsistent findings on healthcare resource utilisation. Unlike Mueller $e t a l^{30}$ Kwan et $a l^{31}$ did not report significant association between post-hospital healthcare utilisation and medication discrepancies identified through medication reconciliation interventions. Both reviews broadly assessed the effect of medication reconciliation produced by various strategies, including the use of collaborative models. The aim of the present review was, thus, to specifically assess the effectiveness of pharmacist-led medication reconciliation programmes on clinical outcomes during the transition to and from hospital settings.

\section{METHODS}

\section{Data sources and searches}

The study was conducted utilising Preferred Reporting Items for Systematic Reviews and Meta-Analyses (PRISMA) group guidelines, ${ }^{32}$ including the PRISMA checklist, to ensure inclusion of relevant information. An initial limited search of articles was undertaken and the search strategy was broadened after analysis of the text words contained in the title, abstract and index terms. 'Medication reconciliation', 'medication discrepancies', 'medication errors', 'medication history' and 'pharmac*', were the main Medicine Subject Headings $(\mathrm{MeSH})$ and text word terms in the electronic searches. Then, we carried out a comprehensive search involving all the collections in the databases until December 2014: PubMed/MEDLINE (1946), Ovid/MEDLINE (1946), International Pharmaceutical Abstracts (1970), EMBASE (1966), PsycINFO (1890) and CINHAL (1937) (see online supplementary appendix A). The reference lists of review articles and included studies were manually searched to locate articles that were not identified in the database search. Article search was performed by one reviewer (ABM) with the support of a medical librarian.

\section{Study selection}

To be included in the selection, studies were required to present the following: papers that reported medication reconciliation intervention primarily and that provide data on any of these clinical end points (all-cause readmission, emergency department (ED) visits, composite rate of readmission and/or ED visits, mortality, adverse drug event (ADE)-related hospital visit). We adopted the definition of 'medication reconciliation' utilised by the Institute for Healthcare Improvement: 'the process of identifying the most accurate list of a patient's current medicines including the name, dosage, frequency and route-and comparing them to the current list in use, recognising and documenting any discrepancies, thus resulting in a complete list of medications'. ${ }^{1}$ Included studies had to be original peer-reviewed research articles that were published in English. The included interventions had to start in the hospital and be performed primarily by a pharmacist, with the aim of improving care transitions to and from a hospital. The intervention had to have been compared with another group that received usual or standard care. 'Usual or standard care' was defined as any care where targeted medication reconciliation was not undertaken as an intervention, or where, if an intervention was conducted, it was not provided by a pharmacist. Along with duplicate references, and other studies that did not satisfy the inclusion criteria and were not medication reconciliation studies, we excluded the following types of studies: other medication reconciliation practices (eg, nurse-led) or practices as part of a multicomponent intervention (eg, medication therapy management), case studies, systematic reviews, qualitative outcomes and non-research articles. Abstracts from conferences and full-texts without raw data available for retrieval were not considered. Therefore, the studies selected for inclusion and exclusion assessment were randomised controlled trials (RCTs), quasi-experimental studies with a control group, and before-and-after studies that evaluated pharmacist-led medication reconciliation programmes at hospital transitions. The titles and abstracts were screened by one author (ABM), and studies identified for full-text review and selected according to inclusion criteria were agreed on by the second (AJM) and third reviewer (JEB).

\section{Data extraction}

One review author (ABM) was responsible for data extraction from full-texts, using a modified adopted Cochrane EPOC data collection checklist, ${ }^{33}$ including quality assessment of studies. The following information was extracted from each included study: name of first author, year of publication, country and setting where the study was conducted, study design, sample size, target of intervention, patient characteristics, components of intervention, and relevant outcomes and results. If insufficient details were reported, study authors were contacted for further information.

\section{Outcomes and statistical analysis}

Our analysis included studies that reported at least one of these end points: healthcare utilisation (readmission, ED visit and composite readmission, and/or ED visit), 
mortality and ADE-related hospital visits, compared with usual care in the other arm; and using at least 30 days of follow-up. Studies were eligible for metaanalysis if such end point could be extractable. We analysed data in accordance with the Cochrane handbook. $^{34}$ Together with $95 \%$ CIs for each outcome, we derived the relative risk and weighted mean differences for dichotomous and continuous variables, respectively.

After we combined data, the analyses were conducted with Cochrane Review Manager (RevMan) V.5.3 software (Copenhagen: The Nordic Cochrane Centre, The Cochrane Collaboration, 2014). We performed separate analyses for each outcome measured compared with usual care. We synthesised the results by constructing a forest plot using a random effects model for each of the outcomes. We analysed intention-to-treat data whenever available. The Mantel-Haenszel risk ratio (RR) summary estimate was determined for outcome measures of dichotomous variables and the weighted mean difference was calculated for continuous data variables. To confirm the reliability of the summary estimate, $95 \%$ CIs were calculated. Since the analyses included medication reconciliation interventions with multiple components, different designs and follow-up periods, we set a priori that might be associated with some variation in the outcomes between the studies. When there were at least five studies per outcome, subgroup analyses were carried out according to methodological design factors (RCT and non-randomised studies) and outcome timing (duration of follow-up). For studies that reported outcomes at a different duration, the longer follow-up period was taken in the analysis, if there was no difference in the summary estimate. Otherwise, meta-analysis was performed separately for the long-duration and shortduration subgroups. We assessed statistical heterogeneity among studies through calculating $\tau^{2}, \chi^{2}(\mathrm{Q}), \mathrm{I}^{2}$ and $\mathrm{p}$ value. We conducted sensitivity analysis to check the stability of summary estimates to outliers and the change in $\mathrm{I}^{2}$ when any of the studies were withdrawn from the analysis. We evaluated publication bias by inspection of funnel plot, and Begg-Mazumdar and Egger's test using Comprehensive Meta-analysis, V.3 (Biostat, Englewood, New Jersey, USA). In all analyses, $p$ value $<0.05$ was considered as statistically significant.

We assessed the risk of bias of individual studies with EPOC risk of bias tool. ${ }^{33}$ The main domains considered were random sequence generation, allocation concealment, blinding of outcome assessment, attrition and reporting biases. We also determined whether groups were balanced at baseline in terms of characteristics and outcomes. Included studies were evaluated for each domain and a quality scoring was then calculated for each study. Studies with 'clear data' on each of the domains were given a score of 1 , and studies were assigned a point score out of the maximum of 9 (9 domains were included in the risk of bias assessment).
RESULTS

\section{Identification and selection of studies}

We identified a total of 2551 citations from searches in the electronic databases and 59 additional records were identified in reference lists of included studies. After removal of duplicate records, title and abstract screening was applied on 1832 publications. After title and abstract review, 1731 publications did not meet the inclusion criteria-the focus for the majority of studies was not related to medication reconciliation interventions. The remaining 101 publications were obtained in full-text and assessed for inclusion. Most full-text articles were excluded either due to reporting of a different outcome of interest $(n=34)$ or because medication reconciliation was not the primary intervention $(n=11)$ (see online supplementary appendix B). After applying all the inclusion criteria, we finally included 17 articles (figure 1).

\section{Characteristics of included studies}

Major characteristics of the included studies are presented in table 1 . They were randomised controlled trials $(n=8,47 \%)$, before-and-after studies $(n=6,35 \%)$ and non-randomised controlled trials $(n=3,18 \%)$. The majority of the studies were conducted in the USA (11 studies), ${ }^{35-45}$ and the remainder were in Sweden (3 studies), ${ }^{46-48}$ Ireland (2 studies) ${ }^{49}{ }^{50}$ and Australia (1 study). ${ }^{51}$ The studies had been conducted between 2002 and 2014. The included studies involved a total of 21342 adult patients of various ages with sample sizes ranging from 41 to 8959 individuals. No studies in paediatrics were identified. Only three studies were confined to multicentre. ${ }^{38} 4951$ Most studies reported outcomes up to 30 days of follow-up after selection of eligible patients; only six studies $^{37}{ }^{46-50}$ reported longer follow-up of 3-month or more. Interventions were initiated at different care transitions; most were conducted at multiple transitions, ${ }^{35} 37-40424446-51$ and all studies targeting a single transition intervention were carried out at hospital discharge. ${ }^{36} 414345$

Most studies recruited high-risk patients (including elderly patients, patients with multiple medications and patients at risk of medication-related events). Five studies $36 \quad 373944 \quad 48$ focused on a specific patient population, mainly patients with heart failure and chronic obstructive pulmonary disease (COPD). Methodologically, one study ${ }^{35}$ stratified patients into two groups: general population and high-risk patients, and another study ${ }^{37}$ randomised the population into two levels of intervention: minimal and enhanced.

Some studies compared comprehensive medication reconciliation programmes, for example, multifaceted interventions including telephone follow-up and/or home visit, ${ }^{44} 4851$ and patient counselling, ${ }^{35} 384145$ or both telephone/home visit and patient counselling. ${ }^{37} \quad 40 \quad 42 \quad 43 \quad 46 \quad 4950$ After medication reconciliation, a few studies $^{42}$ 46-49 additionally included a formal medication review. Comparator groups in the 
Figure 1 PRISMA flow diagram of the selection of eligible studies.

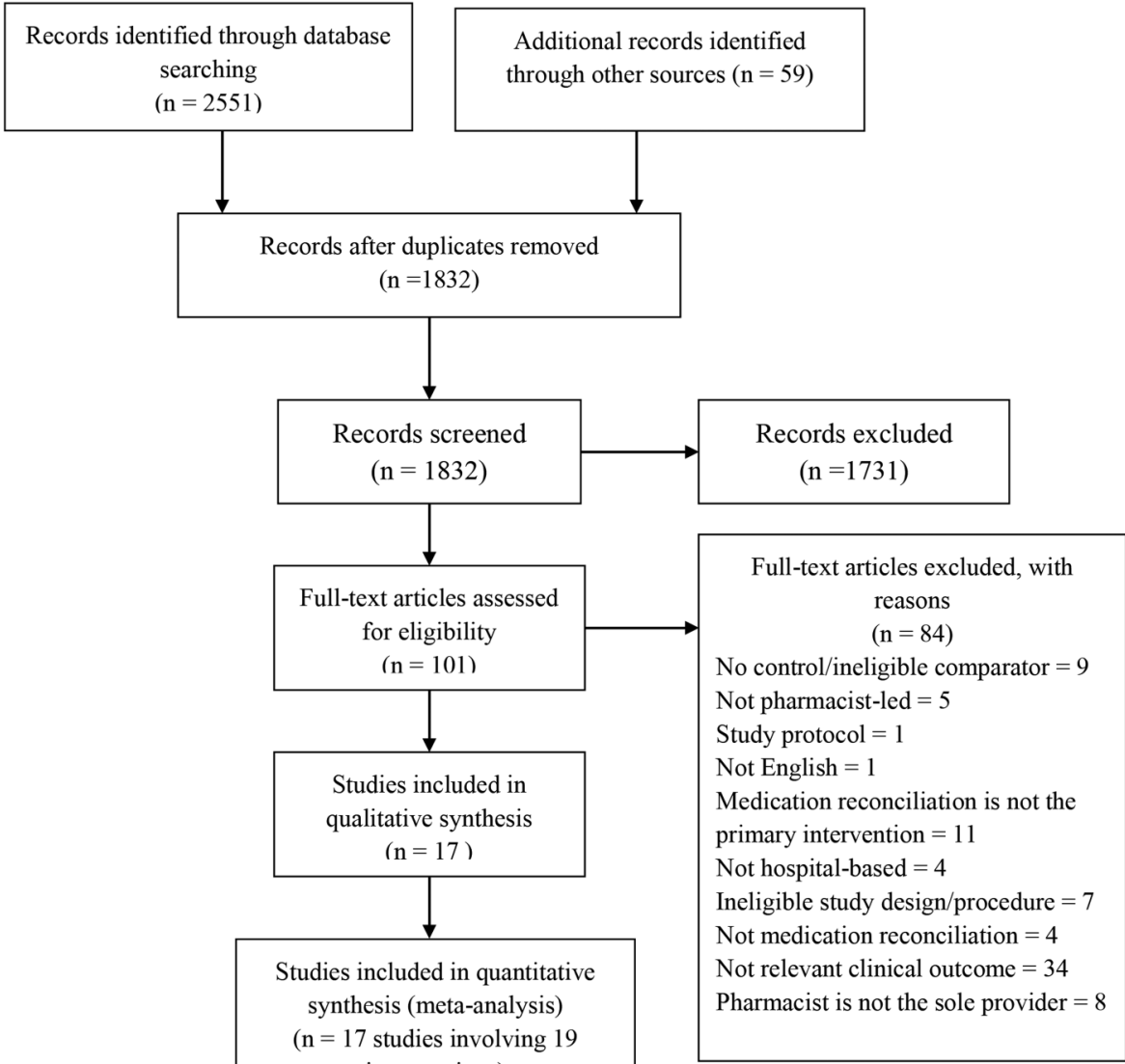

interventions) included studies were varied, and most studies compared medication reconciliation interventions with a usual care group that did not receive pharmacist-led intervention.

\section{Risk of bias assessment}

Patients included in the study were similar in baseline characteristics except in five studies, ${ }^{36} 38394548$ which were not clear or different in patient characteristics. However, in only three studies ${ }^{43} 4851$ were baseline clinical outcomes reported or was some form of adjustment analysis performed. Eight out of 17 studies $393940424649-51$ provided enough details on randomisation procedure to be judged as adequate. Among these studies, allocation concealment was fully described in all reports except one. ${ }^{51}$ In all but three studies ${ }^{43} 4550$ had care providers and outcome assessors been blinded or objective health outcomes reported. Five studies ${ }^{37} 41474851$ achieved more than $80 \%$ complete follow-up. However, only a few studies examined the impact of losses to follow-up or drop-out. High-risk of contamination was suspected in four studies. ${ }^{35} 374147$ At least one of our outcomes of interest was selectively reported in four studies. ${ }^{36} 49-51$ Overall, on a scale of 9, quality of randomised controlled trials falls within a range of 4-8, whereas for non-randomised controlled trials a lower range of $1-5$ score was attained (see online supplementary appendix $\mathrm{C}$ ).

\section{Effect of interventions}

Of the 14 studies that reported data on all-cause readmissions, 13 were eligible for meta-analysis. One study ${ }^{35}$ measured this outcome for a high-risk population separately; and another study ${ }^{37}$ reported it for two different interventions. Thus, 15 interventions were meta-analysed. Eight studies reported this outcome at 30 days, ${ }^{35} \quad 36 \quad 3941 \quad 43-45 \quad 51$ while three ${ }^{46} 48 \quad 49$ reported long-term data and two studies ${ }^{37} 38$ reported both. Seven studies ${ }^{35} \quad \begin{array}{lllllll}38 & 39 & 41 & 44 & 45 & 49 & \text { showed a significant }\end{array}$ reduction $(p<0.05)$ in rehospitalisations although two $^{39} 44$ of them had a very small sample size. The pooled RR ( $\mathrm{n}=21969$ patients) across all studies was 0.81 (95\% CI 0.70 to 0.95$)$. However, the results of these studies for this end point are substantially heterogeneous (figure 2A). With regard to all-cause emergency department (ED) contacts, seven of eight studies $^{35} \quad 37-3943 \quad 46 \quad 48$ that measured ED visit as an outcome were pooled. Considering studies that gave two sets of data, nine interventions were meta-analysed. The pooled analysis across all interventions showed some significant difference between the intervention and usual care (RR $0.72 ; 95 \%$ CI 0.57 to 0.92 ; figure 2B). Evidence showed extreme heterogeneity in this outcome; however, the findings were different when the study by Gardella et $a \vec{l}^{8}$ was removed; there was no heterogeneity without affecting the significance difference $\left(p=0.25 ; I^{2}=22 \%\right.$, RR $0.89 ; 95 \%$ CI 0.79 to 0.99$)$. 


\begin{tabular}{|c|c|c|c|c|c|c|c|c|c|c|c|c|}
\hline $\begin{array}{l}\text { Author, } \\
\text { Year }\end{array}$ & $\begin{array}{l}\text { Country, } \\
\text { Setting }\end{array}$ & $\begin{array}{l}\text { Study } \\
\text { design }\end{array}$ & Intervention & Comparator & $\begin{array}{l}\text { Target of } \\
\text { intervention }\end{array}$ & Inclusion & Exclusion & $\begin{array}{l}\text { Components of } \\
\text { intervention }\end{array}$ & Comparator & $\begin{array}{l}\text { Follow-up } \\
\text { Period }\end{array}$ & $\begin{array}{l}\text { Relevant } \\
\text { outcomes }\end{array}$ & Main results \\
\hline $\begin{array}{l}\text { Anderegg } \\
\text { et al } 2014^{35}\end{array}$ & $\begin{array}{l}\text { USA, } \\
\text { single } \\
\text { centre }\end{array}$ & $\begin{array}{l}\text { Before- } \\
\text { after }\end{array}$ & 1664 & 1652 & $\begin{array}{l}\text { Admission, } \\
\text { discharge }\end{array}$ & $\begin{array}{l}\text { Age } 18 \text { years or older, } \\
\text { discharge from } \\
\text { internal medicine, } \\
\text { family medicine, } \\
\text { cardiology, or } \\
\text { orthopaedic surgery } \\
\text { medical }\end{array}$ & $\begin{array}{l}\text { Mental illness/ } \\
\text { alcohol or drug use; } \\
\text { discharge to a } \\
\text { rehabilitation unit/ } \\
\text { long-term care } \\
\text { facility, readmission } \\
\text { for chemotherapy/ } \\
\text { radiation therapy/ } \\
\text { rehabilitation } \\
\text { therapy }\end{array}$ & $\begin{array}{l}\text { Admission MedRec, } \\
\text { Discharge MedRec, } \\
\text { patient education, } \\
\text { medication calendar }\end{array}$ & $\begin{array}{l}\text { Control group } \\
\text { (admission } \\
\text { MedRec as } \\
\text { needed) }\end{array}$ & 30 days & $\begin{array}{l}\text { Readmission, } \\
\text { Readmission } \\
\text { and/or ED visit }\end{array}$ & $\begin{array}{l}\text { 30-day readmission } \\
\text { and/or ED visit } \\
\text { (general population): } \\
\text { NS; } 30 \text {-day } \\
\text { readmission } \\
\text { (high-risk): } 12.3 \% \text { (I) } \\
\text { vs } 17.8 \%(\mathrm{U}), \mathrm{p}=0.042\end{array}$ \\
\hline $\begin{array}{l}\text { Bolas et al } \\
2004^{50}\end{array}$ & $\begin{array}{l}\text { Ireland, } \\
\text { single } \\
\text { centre }\end{array}$ & $\mathrm{RCT}$ & 81 & 81 & $\begin{array}{l}\text { Inpatient stay, } \\
\text { discharge, } \\
\text { postdischarge }\end{array}$ & $\begin{array}{l}\text { Age } 55 \text { years or older, } \\
\text { at least } 3 \text { regular } \\
\text { medications }\end{array}$ & $\begin{array}{l}\text { Transfer to another } \\
\text { hospital or nursing } \\
\text { home, unable to } \\
\text { communicate, } \\
\text { mental illness or } \\
\text { alcohol-related } \\
\text { admission, follow-up } \\
\text { was declined }\end{array}$ & $\begin{array}{l}\text { Medication liaison } \\
\text { service } \\
\text { (comprehensive } \\
\text { medication history, } \\
\text { discharge letter } \\
\text { faxed to GP and } \\
\text { community } \\
\text { pharmacist, } \\
\text { medicines record } \\
\text { sheet, discharge } \\
\text { counselling, home } \\
\text { visit/telephone call) }\end{array}$ & $\begin{array}{l}\text { Standard clinical } \\
\text { pharmacy } \\
\text { service (not } \\
\text { include } \\
\text { discharge } \\
\text { counselling and } \\
\text { liaison service) }\end{array}$ & 3 month & $\begin{array}{l}\text { Readmission, } \\
\text { hospital stay } \\
\text { (following } \\
\text { readmission) }\end{array}$ & $\begin{array}{l}\text { Readmission rate: } \\
p>0.05 \text {; Length of stay: } \\
p>0.05\end{array}$ \\
\hline $\begin{array}{l}\text { Eisenhower } \\
2014^{36}\end{array}$ & $\begin{array}{l}\text { US, single } \\
\text { centre }\end{array}$ & $\begin{array}{l}\text { Before- } \\
\text { after }\end{array}$ & 25 & 60 & Discharge & $\begin{array}{l}\text { Age } 65 \text { years or older, } \\
\text { with history of COPD }\end{array}$ & $\begin{array}{l}\text { Left the hospital } \\
\text { without medical } \\
\text { advice, death within } \\
30 \text { days of } \\
\text { discharge }\end{array}$ & $\begin{array}{l}\text { MedRec at } \\
\text { discharge, } \\
\text { Medication } \\
\text { reconciliation form, } \\
\text { discharge summary }\end{array}$ & $\begin{array}{l}\text { Usual care } \\
\text { (pharmacist was } \\
\text { not present } \\
\text { during baseline } \\
\text { data collection) }\end{array}$ & 30 days & Readmission & $\begin{array}{l}\text { Readmission rate: } 16 \% \\
\text { (I) vs } 22.2 \% \text { (U) }\end{array}$ \\
\hline $\begin{array}{l}\text { Farris et al } \\
2014^{37}\end{array}$ & $\begin{array}{l}\text { USA, } \\
\text { Single } \\
\text { centre }\end{array}$ & $\mathrm{RCT}$ & $\begin{array}{l}\text { Minimal }=312 \\
\text { Enhanced }=311\end{array}$ & 313 & $\begin{array}{l}\text { Admission, } \\
\text { inpatient stay, } \\
\text { discharge }\end{array}$ & $\begin{array}{l}18 \text { years or older, } \\
\text { English or Spanish } \\
\text { speaker, diagnosis of } \\
\text { HPN, } \\
\text { hyperlipidaemia, HF, } \\
\text { CAD, MI, stroke, TIA, } \\
\text { asthma, COPD or } \\
\text { receiving oral } \\
\text { anticoagulation }\end{array}$ & $\begin{array}{l}\text { Admission to } \\
\text { psychiatry, surgery } \\
\text { or haematology/ } \\
\text { oncology service, } \\
\text { could not use a } \\
\text { telephone, had life } \\
\text { expectancy } \\
<6 \text { months, had } \\
\text { dementia or } \\
\text { cognitive } \\
\text { impairment }\end{array}$ & $\begin{array}{l}\text { Admission MedRec, } \\
\text { patient education } \\
\text { during inpatient stay, } \\
\text { discharge } \\
\text { counselling, } \\
\text { discharge } \\
\text { medication list, } \\
\text { telephone call, care } \\
\text { plan faxed to primary } \\
\text { care physician/ } \\
\text { community } \\
\text { pharmacist }\end{array}$ & $\begin{array}{l}\text { Usual care } \\
\text { (admission } \\
\text { MedRec, } \\
\text { nurse-led } \\
\text { discharge } \\
\text { counselling and } \\
\text { medication list) }\end{array}$ & 90 days & $\begin{array}{l}\text { ADEs, } \\
\text { readmission, } \\
\text { ED visit, } \\
\text { readmission } \\
\text { and/or ED visit }\end{array}$ & $\begin{array}{l}16 \% \text { experienced an } \\
\mathrm{AE}, \text { Healthcare } \\
\text { utilisation at } 30 \text { days } \\
\text { and } 90 \text { days: NS }\end{array}$ \\
\hline
\end{tabular}




\begin{tabular}{|c|c|c|c|c|c|c|c|c|c|c|c|c|}
\hline $\begin{array}{l}\text { Author, } \\
\text { Year }\end{array}$ & $\begin{array}{l}\text { Country, } \\
\text { Setting }\end{array}$ & $\begin{array}{l}\text { Study } \\
\text { design }\end{array}$ & Intervention & Comparator & $\begin{array}{l}\text { Target of } \\
\text { intervention }\end{array}$ & Inclusion & Exclusion & $\begin{array}{l}\text { Components of } \\
\text { intervention }\end{array}$ & Comparator & $\begin{array}{l}\text { Follow-up } \\
\text { Period }\end{array}$ & $\begin{array}{l}\text { Relevant } \\
\text { outcomes }\end{array}$ & Main results \\
\hline $\begin{array}{l}\text { Gardella } \\
\text { et al } 2012^{38}\end{array}$ & $\begin{array}{l}\text { US, } \\
\text { multicentre }\end{array}$ & $\begin{array}{l}\text { Before- } \\
\text { after }\end{array}$ & 1624 & 7335 & $\begin{array}{l}\text { Preadmission } \\
\text { to post } \\
\text { discharge }\end{array}$ & NA & NA & $\begin{array}{l}\text { Preadmission } \\
\text { medication list, } \\
\text { patient education }\end{array}$ & $\begin{array}{l}\text { Historical control } \\
\text { group } \\
\text { (preadmission } \\
\text { medication list } \\
\text { gathered by } \\
\text { nurse) }\end{array}$ & 60 days & $\begin{array}{l}\text { ADE, ED visits } \\
\text { and } \\
\text { readmission }\end{array}$ & $\begin{array}{l}\text { 30-day readmission: } \\
6 \%(\mathrm{I}) \text { vs } 13.1 \%(\mathrm{U}) \\
\text { (OR } 2.34,95 \% \mathrm{Cl} 1.87 \\
\text { to } 2.94, \mathrm{p}<0.001 \text { ); } \\
60 \text {-day readmission: } \\
2.7 \% \text { (I) vs } 7.7 \%(\mathrm{U}) \\
\text { (OR } 3.02,95 \% \mathrm{Cl} 2.18 \\
\text { to } 4.19, \mathrm{p}<0.001 \text { ) }\end{array}$ \\
\hline $\begin{array}{l}\text { Gillespie } \\
\text { et al } 2009^{46}\end{array}$ & $\begin{array}{l}\text { Sweden, } \\
\text { single } \\
\text { centre }\end{array}$ & RCT & 182 & 186 & $\begin{array}{l}\text { Admission, } \\
\text { inpatient stay } \\
\text { and discharge }\end{array}$ & Age 80 or older & $\begin{array}{l}\text { Previous admission } \\
\text { during the study } \\
\text { period }\end{array}$ & $\begin{array}{l}\text { Admission MedRec, } \\
\text { discharge } \\
\text { counselling, } \\
\text { medication review, } \\
\text { faxing discharge } \\
\text { summary to primary } \\
\text { care physicians, } \\
\text { telephone follow-up } \\
\text { at } 2 \text { months }\end{array}$ & $\begin{array}{l}\text { Usual care } \\
\text { (without } \\
\text { pharmacist } \\
\text { involvement) }\end{array}$ & 12 month & $\begin{array}{l}\text { Readmissions, } \\
\text { ED visits, } \\
\text { mortality }\end{array}$ & $\begin{array}{l}\text { Readmissions: } 58.2 \% \\
\text { (I) vs } 59.1 \% \text { (U) (OR } \\
0.96,95 \% \mathrm{Cl} 0.64 \text { to } \\
\text { 1.4); ED visits per } \\
\text { patient: } 0.35 \text { (I) vs } 0.66 \\
\text { (U) (OR } 0.53,95 \% \mathrm{Cl} \\
0.37 \text { to } 0.75 \text { ) }\end{array}$ \\
\hline $\begin{array}{l}\text { Hawes et al } \\
2014^{39}\end{array}$ & $\begin{array}{l}\text { US, single } \\
\text { centre }\end{array}$ & RCT & 24 & 37 & $\begin{array}{l}\text { Discharge and } \\
\text { post discharge }\end{array}$ & $\begin{array}{l}\text { High-risk patients ( } \\
\text { HF, COPD, } \\
\text { hyperglycaemic crisis, } \\
\text { stroke ,NSTEM, more } \\
\text { than } 3 \text { hospitalisations } \\
\text { in the past } 5 \text { years., } 8 \\
\text { or more medications } \\
\text { on discharge) }\end{array}$ & $\begin{array}{l}\text { Age }<18 \text { years, } \\
\text { inability to } \\
\text { communicate in } \\
\text { English, unable to } \\
\text { follow-up (no } \\
\text { transportation and } \\
\text { no telephone } \\
\text { access), transfer to } \\
\text { facilities other than } \\
\text { primary care, } \\
\text { decisional } \\
\text { impairment, } \\
\text { incarceration }\end{array}$ & $\begin{array}{l}\text { Post discharge } \\
\text { medication } \\
\text { reconciliation }\end{array}$ & $\begin{array}{l}\text { Usual care (with } \\
\text { no pharmacist } \\
\text { intervention) }\end{array}$ & 30 days & $\begin{array}{l}\text { Readmission, } \\
\text { ED visit, } \\
\text { readmission } \\
\text { and /or ED visit }\end{array}$ & $\begin{array}{l}\text { ED visit: } 0 \text { (I) vs } \\
29.7 \%(U), p=0.004 \text {; } \\
\text { Readmission: } 0 \text { (I) vs } \\
32.4 \%(U), p=0.002 \text {; } \\
\text { Composite of } \\
\text { hospitalisation or ED } \\
\text { visit: } 0 \text { (I) vs } 40.5 \% \\
(C), p<0.001\end{array}$ \\
\hline $\begin{array}{l}\text { Hellstrom } \\
\text { et al } 2011^{47}\end{array}$ & $\begin{array}{l}\text { Sweden, } \\
\text { single } \\
\text { centre }\end{array}$ & $\begin{array}{l}\text { Before- } \\
\text { after }\end{array}$ & 109 & 101 & $\begin{array}{l}\text { Admission, } \\
\text { inpatient stay, } \\
\text { discharge }\end{array}$ & $\begin{array}{l}\text { Age } 65 \text { years or older, } \\
\text { at least one regular } \\
\text { medication }\end{array}$ & $\begin{array}{l}\text { Staying during the } \\
\text { implementation } \\
\text { period }\end{array}$ & $\begin{array}{l}\text { LIMM model, } \\
\text { admission and } \\
\text { discharge MedRec, } \\
\text { medication review } \\
\text { and monitoring, } \\
\text { quality control of } \\
\text { discharge MedRec }\end{array}$ & $\begin{array}{l}\text { Standard care } \\
\text { (no formal } \\
\text { MedRec by } \\
\text { clinical } \\
\text { pharmacists) }\end{array}$ & 3 month & $\begin{array}{l}\text { Readmission } \\
\text { and ED visit, } \\
\text { ADE-related } \\
\text { hospital visit }\end{array}$ & $\begin{array}{l}\text { ED visit and } \\
\text { readmission: } 45 / 108 \text { (I) } \\
\text { vs } 41 / 100(\mathrm{U}) \\
\text { Mortality, } 3 \text { month: } 9 / \\
108 \text { (I) vs } 9 / 100(\mathrm{U}) \\
\text { ADE-related revisit: } 6 / \\
108 \text { (I) vs } 12 / 100(\mathrm{U})\end{array}$ \\
\hline $\begin{array}{l}\text { Hellstrom } \\
\text { et al } 2012^{48}\end{array}$ & $\begin{array}{l}\text { Sweden, } \\
\text { single } \\
\text { centre }\end{array}$ & $\begin{array}{l}\text { Before- } \\
\text { after }\end{array}$ & 1216 & 2758 & $\begin{array}{l}\text { Admission, } \\
\text { inpatient stay }\end{array}$ & $\begin{array}{l}\text { High-risk patients } \\
\text { (age } \geq 65 \text { years with } \\
\text { any of HF, RF) }\end{array}$ & NA & $\begin{array}{l}\text { Admission MedRec, } \\
\text { structured } \\
\text { medication reviews, }\end{array}$ & $\begin{array}{l}\text { Usual care (no } \\
\text { clinical } \\
\text { pharmacists }\end{array}$ & 6 month & $\begin{array}{l}\text { ED visits, } \\
\text { hospital }\end{array}$ & $\begin{array}{l}\text { ED visit: } 48.8 \% \text { (I) vs } \\
51.3 \% \text { (U) (HR 0.95, } \\
95 \% \mathrm{Cl} 0.86 \text { to } 1.04) ;\end{array}$ \\
\hline
\end{tabular}




\begin{tabular}{|c|c|c|c|c|c|c|c|c|c|c|c|c|}
\hline $\begin{array}{l}\text { Author, } \\
\text { Year }\end{array}$ & $\begin{array}{l}\text { Country, } \\
\text { Setting }\end{array}$ & $\begin{array}{l}\text { Study } \\
\text { design }\end{array}$ & Intervention & Comparator & $\begin{array}{l}\text { Target of } \\
\text { intervention }\end{array}$ & Inclusion & Exclusion & $\begin{array}{l}\text { Components of } \\
\text { intervention }\end{array}$ & Comparator & $\begin{array}{l}\text { Follow-up } \\
\text { Period }\end{array}$ & $\begin{array}{l}\text { Relevant } \\
\text { outcomes }\end{array}$ & Main results \\
\hline & & & & & & & & $\begin{array}{l}\text { follow-up at least two } \\
\text { times a week }\end{array}$ & $\begin{array}{l}\text { working in the } \\
\text { wards) }\end{array}$ & & $\begin{array}{l}\text { admissions and } \\
\text { mortality }\end{array}$ & $\begin{array}{l}\text { All ED visits, } \\
\text { hospitalisation or } \\
\text { death: } 58.9 \% \text { (I) vs } \\
61.2 \% \text { (U) (HR } 0.96 \text {, } \\
95 \% \mathrm{Cl} 0.88 \text { to } 1.04) \\
\text { Mortality: } 18.2 \% \text { (I) vs } \\
17.3 \% \text { (U), p=0.55 }\end{array}$ \\
\hline $\begin{array}{l}\text { Koehler } \\
\text { et al } 2009^{40}\end{array}$ & $\begin{array}{l}\text { US, single } \\
\text { centre }\end{array}$ & $\mathrm{RCT}$ & 20 & 21 & $\begin{array}{l}\text { Admission, } \\
\text { discharge and } \\
\text { post discharge }\end{array}$ & $\begin{array}{l}\text { Age } 70 \text { years or older, } \\
\geq 5 \text { medications, } \geq 3 \\
\text { chronic comorbid } \\
\text { conditions, assisted } \\
\text { living, English } \\
\text { language, phone } \\
\text { contact }\end{array}$ & $\begin{array}{l}\text { Primarily surgical } \\
\text { procedure, life } \\
\text { expectancy } \\
\leq 6 \text { months, } \\
\text { residence in } \\
\text { long-term care } \\
\text { facility, refusal to } \\
\text { participate, not } \\
\text { enrolled within } 72 \mathrm{~h} \text {. }\end{array}$ & $\begin{array}{l}\text { Targeted care } \\
\text { bundle, medication } \\
\text { reconciliation and } \\
\text { education, follow-up } \\
\text { call, enhanced } \\
\text { discharge form }\end{array}$ & $\begin{array}{l}\text { Usual care } \\
\text { (nurse and care } \\
\text { coordination } \\
\text { staff providing } \\
\text { care) }\end{array}$ & 60 days & $\begin{array}{l}\text { Readmission } \\
\text { and/or ED visits }\end{array}$ & $\begin{array}{l}30 \text { days readmission/ } \\
\text { ED visits: } 2 / 20 \text { (I) vs } 8 / \\
21(\mathrm{U}), p=0.03 \text {; } \\
60 \text { days readmission/ } \\
\text { ED visits: } 6 / 20 \text { (I) vs } 9 / \\
21 \text { (U), } p=0.52\end{array}$ \\
\hline $\begin{array}{l}\text { Pal et al } \\
2013^{41}\end{array}$ & $\begin{array}{l}\text { US, single } \\
\text { centre }\end{array}$ & NRCT & 537 & 192 & Discharge & $\begin{array}{l}\text { Age } 18 \text { years or older, } \\
\text { at least } 10 \text { regular } \\
\text { medications }\end{array}$ & NA & $\begin{array}{l}\text { Patient counselling, } \\
\text { pharmacist } \\
\text { medication } \\
\text { reconciliation, } \\
\text { medication calendar }\end{array}$ & $\begin{array}{l}\text { Usual care } \\
\text { (without } \\
\text { discharge review } \\
\text { by pharmacist) }\end{array}$ & 30 days & Readmission & $\begin{array}{l}30 \text { days readmission: } \\
16.8 \% \text { (I) vs } 26.0 \% \\
\text { (U), } p=0.006 \\
\text { ADE prevented: } 52.8 \%\end{array}$ \\
\hline $\begin{array}{l}\text { Schnipper } \\
\text { et al } 2006^{42}\end{array}$ & $\begin{array}{l}\text { US, single } \\
\text { centre }\end{array}$ & $\mathrm{RCT}$ & 92 & 84 & $\begin{array}{l}\text { Inpatient stay, } \\
\text { discharge, } \\
\text { post discharge }\end{array}$ & $\begin{array}{l}\text { Discharge to home, } \\
\text { contacted } 30 \text { days } \\
\text { after discharge, spoke } \\
\text { English, cared for } \\
\text { primary care } \\
\text { physician/internal } \\
\text { medicine resident }\end{array}$ & NA & $\begin{array}{l}\text { Discharge } \\
\text { medication } \\
\text { reconciliation, } \\
\text { telephone follow-up, } \\
\text { medication review, } \\
\text { standard email } \\
\text { template, patient } \\
\text { counselling }\end{array}$ & $\begin{array}{l}\text { Usual care } \\
\text { (medication } \\
\text { review by a } \\
\text { pharmacist and } \\
\text { discharge } \\
\text { counselling by a } \\
\text { nurse) }\end{array}$ & & $\begin{array}{l}\text { ADEs-related } \\
\text { hospital visit, } \\
\text { readmission } \\
\text { and/or ED visit }\end{array}$ & $\begin{array}{l}\text { Preventable ADE: } 1 \% \\
\text { (I) vs } 11 \%(\mathrm{U}), \mathrm{p}=0.01 \text {; } \\
\text { ED visit/readmission: } \\
30 \% \text { (I) vs } 30 \%(\mathrm{U}) \text {, } \\
\mathrm{p}>0.99 ; \text { preventable } \\
\text { medication-related } \\
\text { healthcare utilisation: } \\
1 \%(\mathrm{I}) \text { vs } 8 \%(\mathrm{U}), \mathrm{p}= \\
0.03\end{array}$ \\
\hline $\begin{array}{l}\text { Scullin et al } \\
2007^{49}\end{array}$ & $\begin{array}{l}\text { Ireland, } \\
\text { multicentre }\end{array}$ & $\mathrm{RCT}$ & 371 & 391 & $\begin{array}{l}\text { Admission, } \\
\text { inpatient stay, } \\
\text { discharge }\end{array}$ & $\begin{array}{l}\text { Age } 65 \text { years or older, } \\
\text { at least } 4 \text { regular } \\
\text { medications, taking } \\
\text { antidepressants, } \\
\text { previous admission in } \\
\text { the past } 6 \text { months, } \\
\text { taking intravenous } \\
\text { antibiotics }\end{array}$ & $\begin{array}{l}\text { Scheduled } \\
\text { admissions and } \\
\text { admissions from } \\
\text { private nursing } \\
\text { homes }\end{array}$ & $\begin{array}{l}\text { Integrated medicines } \\
\text { management service } \\
\text { admission and } \\
\text { discharge MedRec, } \\
\text { inpatient medication } \\
\text { review and } \\
\text { counselling, } \\
\text { telephone follow-up }\end{array}$ & $\begin{array}{l}\text { Usual care (did } \\
\text { not receive } \\
\text { integrated } \\
\text { medicines } \\
\text { management } \\
\text { service) }\end{array}$ & 12 month & $\begin{array}{l}\text { Length of } \\
\text { hospital stay, } \\
\text { readmission }\end{array}$ & $\begin{array}{l}\text { LoS reduced by } 2 \text { days } \\
\text { for intervention vs } \\
\text { usual care, } p=0.003 \\
\text { Readmissions per } \\
\text { patient: } 0.8 \text { (I) vs } 1 \text { (U) }\end{array}$ \\
\hline $\begin{array}{l}\text { Stowasser } \\
\text { et al } 2002^{51}\end{array}$ & $\begin{array}{l}\text { Australia, } \\
\text { multicentre }\end{array}$ & $\mathrm{RCT}$ & 113 & 127 & $\begin{array}{l}\text { Admission, } \\
\text { discharge }\end{array}$ & $\begin{array}{l}\text { Return to the } \\
\text { community following } \\
\text { discharge }\end{array}$ & $\begin{array}{l}\text { Outpatients, } \\
\text { discharge to hostel } \\
\text { or nursing home, }\end{array}$ & $\begin{array}{l}\text { Medication liaison } \\
\text { service-medication } \\
\text { history confirmation }\end{array}$ & $\begin{array}{l}\text { Usual care (no } \\
\text { medication } \\
\text { liaison service) }\end{array}$ & 30 days & $\begin{array}{l}\text { Mortality, } \\
\text { readmission, } \\
\text { ED visit }\end{array}$ & $\begin{array}{l}\text { Mortality, } 30 \text { days: } 2 / \\
113 \text { (I) vs } 3 / 127 \text { (U): } \\
\text { NS }\end{array}$ \\
\hline
\end{tabular}




\begin{tabular}{|c|c|c|c|c|c|c|c|c|c|c|c|c|}
\hline $\begin{array}{l}\text { Author, } \\
\text { Year }\end{array}$ & $\begin{array}{l}\text { Country, } \\
\text { Setting }\end{array}$ & $\begin{array}{l}\text { Study } \\
\text { design }\end{array}$ & Intervention & Comparator & $\begin{array}{l}\text { Target of } \\
\text { intervention }\end{array}$ & Inclusion & Exclusion & $\begin{array}{l}\text { Components of } \\
\text { intervention }\end{array}$ & Comparator & $\begin{array}{l}\text { Follow-up } \\
\text { Period }\end{array}$ & $\begin{array}{l}\text { Relevant } \\
\text { outcomes }\end{array}$ & Main results \\
\hline & & & & & & & $\begin{array}{l}\text { previous enrolment, } \\
\text { unable to provide } \\
\text { consent and } \\
\text { follow-up }\end{array}$ & $\begin{array}{l}\text { with community } \\
\text { healthcare } \\
\text { professionals } \\
\text { (telephone, faxing), } \\
30 \text { days post } \\
\text { follow-up }\end{array}$ & & & & $\begin{array}{l}\text { Readmissions: } 12 / 113 \\
\text { (I) vs } 17 / 127(\mathrm{U}) \\
\text { ED visit per patient: } \\
7.54 \text { (I) vs } 9.94(\mathrm{U})\end{array}$ \\
\hline $\begin{array}{l}\text { Walker et al } \\
2009^{43}\end{array}$ & $\begin{array}{l}\text { US, single } \\
\text { centre }\end{array}$ & NRCT & 138 & 366 & $\begin{array}{l}\text { Discharge, } \\
\text { post discharge }\end{array}$ & $\begin{array}{l}\text { Age } 18 \text { years or older, } \\
5 \text { or more regular } \\
\text { medications, receiving } \\
1 \text { or more targeted } \\
\text { medications, having } 2 \\
\text { or more therapy } \\
\text { modification, unable } \\
\text { to manage their } \\
\text { medication, receiving } \\
\text { a medication requiring } \\
\text { therapeutic drug } \\
\text { monitoring }\end{array}$ & $\begin{array}{l}\text { Non-English } \\
\text { speaking, stay of } \\
21 \text { days or longer }\end{array}$ & $\begin{array}{l}\text { Patient interviews, } \\
\text { follow-up plan, } \\
\text { medication } \\
\text { counselling, } \\
\text { telephone follow-up }\end{array}$ & $\begin{array}{l}\text { Usual care } \\
\text { (nurse-led } \\
\text { service) }\end{array}$ & 30 days & $\begin{array}{l}\text { Readmission, } \\
\text { ED visit, } \\
\text { readmission } \\
\text { and/or ED visit }\end{array}$ & $\begin{array}{l}\text { Readmission, } 14 \text { days: } \\
12.6 \% \text { (I) vs } 11.5 \% \\
\text { (U), } p=0.65 \text {; } \\
\text { Readmission, } 30 \text { days: } \\
22.1 \% \text { (I) vs } 18.0 \% \\
\text { (U), } p=0.17 \text {; } \\
\text { Readmissions and/or } \\
\text { ED visits: } 27.4 \% \text { (I) vs } \\
25.7 \% \text { (U), } p=0.61\end{array}$ \\
\hline $\begin{array}{l}\text { Warden } \\
\text { et al } 2014^{44}\end{array}$ & $\begin{array}{l}\text { US, single } \\
\text { centre }\end{array}$ & $\begin{array}{l}\text { Before- } \\
\text { after }\end{array}$ & 35 & 115 & $\begin{array}{l}\text { Admission, } \\
\text { inpatient stay, } \\
\text { discharge }\end{array}$ & $\begin{array}{l}\text { Age } 18-85 \text { years, } \\
\text { systolic dysfunction } \\
(E F \leq 40)\end{array}$ & $\begin{array}{l}\text { Diastolic } \\
\text { dysfunction, valve } \\
\text { replacement/left } \\
\text { ventricular assist } \\
\text { device }\end{array}$ & $\begin{array}{l}\text { Medication } \\
\text { reconciliation } \\
\text { (admission and } \\
\text { discharge), } \\
\text { discharge } \\
\text { instructions, } \\
\text { telephone follow-up }\end{array}$ & $\begin{array}{l}\text { Historical control } \\
\text { group } \\
\text { (physicians- } \\
\text { admission } \\
\text { MedRec; } \\
\text { nurses- } \\
\text { discharge } \\
\text { counselling) }\end{array}$ & 30 days & Readmission & $\begin{array}{l}\text { All cause readmission, } \\
30 \text {-day : } 17 \% \text { (I) vs } \\
38 \% \text { (U) (RR } 0.45 \text {, } \\
95 \% \mathrm{Cl} 0.21 \text { to } 0.96 \text {, } \\
\mathrm{p}=0.02), 30 \text { days } \\
\text { HF-related } \\
\text { readmission: } 6 \%(\mathrm{I}) \text { vs } \\
18 \% \text { (U) (RR } 0.31 \text {, } \\
95 \% \mathrm{Cl} 0.08 \text { to } 1.27 \text {, } \\
\mathrm{p}=0.11 \text { ) }\end{array}$ \\
\hline $\begin{array}{l}\text { Wilkinson } \\
\text { et al } 2011^{45}\end{array}$ & $\begin{array}{l}\text { US, single } \\
\text { centre }\end{array}$ & NRCT & 229 & 440 & Discharge & $\begin{array}{l}\text { Age } 18 \text { years or older, } \\
\text { English speaking, } \\
\text { patients with } \\
\text { depression, receiving } \\
\text { high-risk medications } \\
\text { and polypharmacy, } \\
\text { poor health literacy, } \\
\text { having an absence of } \\
\text { social support, prior } \\
\text { hospitalisation within } \\
\text { the past } 6 \text { months }\end{array}$ & $\begin{array}{l}\text { Refusal of } \\
\text { pharmacist } \\
\text { education, transfer } \\
\text { to a skilled nursing } \\
\text { facility, or discharge } \\
\text { when the } \\
\text { pharmacist was not } \\
\text { available }\end{array}$ & $\begin{array}{l}\text { Medication history at } \\
\text { admission, during } \\
\text { hospitalisation and } \\
\text { discharge, patient } \\
\text { education on } \\
\text { discharge }\end{array}$ & $\begin{array}{l}\text { Control group } \\
\text { (pharmacists not } \\
\text { provide } \\
\text { medication } \\
\text { counselling at } \\
\text { discharge) }\end{array}$ & 30 days & Readmission & $\begin{array}{l}\text { Readmission rate: } \\
15.7 \%(\mathrm{I}) \text { vs } 21.6 \%(\mathrm{U}) \\
\text { (RR } 0.728,95 \% \mathrm{Cl} \\
0.514 \text { to } 1.032, \mathrm{p} \\
=0.04 \text { ) }\end{array}$ \\
\hline
\end{tabular}


A All-cause readmission

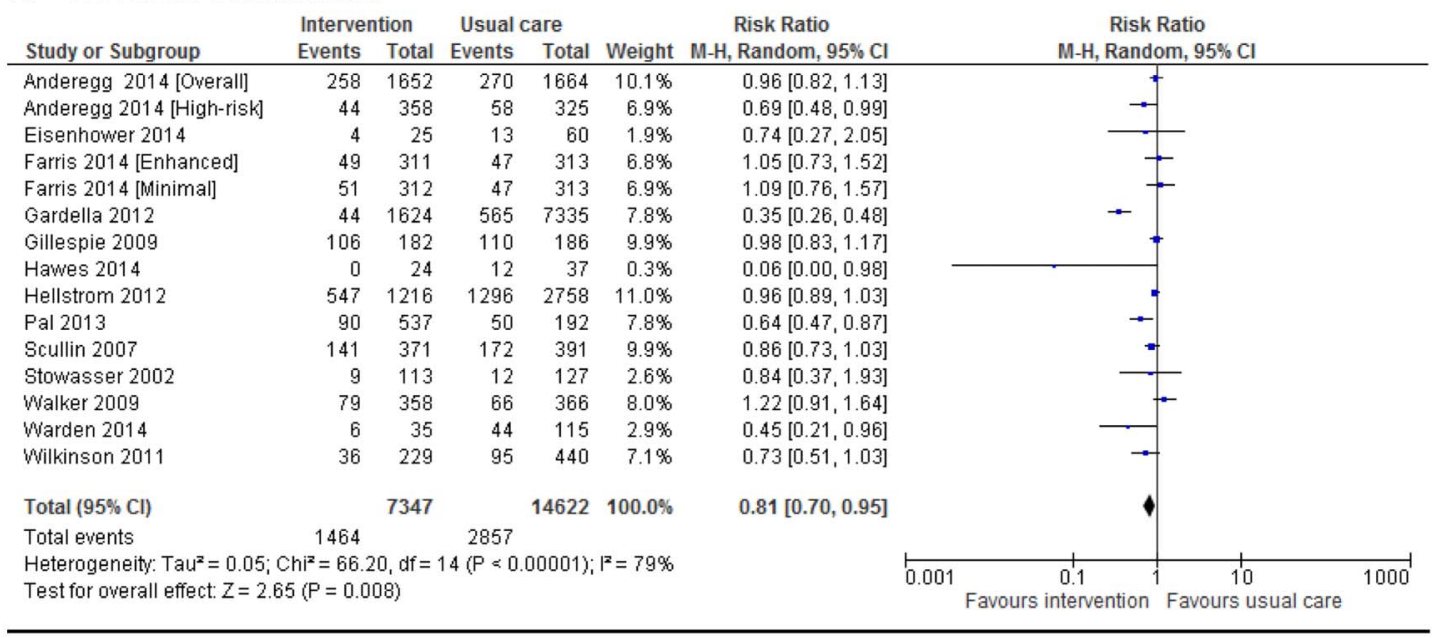

\section{B All-cause emergency department (ED) visits}

\begin{tabular}{|c|c|c|c|c|c|c|c|c|c|c|}
\hline \multirow[b]{2}{*}{ Study or Subgroup } & \multicolumn{2}{|c|}{ Intervention } & \multicolumn{2}{|c|}{ Usual care } & \multicolumn{2}{|r|}{ Risk Ratio } & \multirow{2}{*}{\multicolumn{4}{|c|}{$\begin{array}{c}\text { Risk Ratio } \\
\text { M-H, Random, } 95 \% \mathrm{Cl}\end{array}$}} \\
\hline & Events & Total & Events & Total & Weight & M-H, Random, $95 \% \mathrm{Cl}$ & & & & \\
\hline Anderegg 2014 [Overall] & 155 & 1652 & 168 & 1664 & $15.2 \%$ & $0.93[0.76,1.14]$ & & & & \\
\hline Anderegg 2014 [High-risk] & 22 & 358 & 31 & 325 & $9.4 \%$ & $0.64[0.38,1.09]$ & & & & \\
\hline Farris 2014 [Enhanced] & 41 & 311 & 46 & 313 & $11.8 \%$ & $0.90[0.61,1.33]$ & & & & \\
\hline Farris 2014 [Minimal] & 40 & 312 & 46 & 313 & $11.8 \%$ & $0.87[0.59,1.29]$ & & & & \\
\hline Gardella 2012 & 20 & 1424 & 381 & 7199 & $10.8 \%$ & $0.27[0.17,0.41]$ & & $\rightarrow$ & & \\
\hline Gillespie 2009 & 36 & 182 & 52 & 186 & $12.2 \%$ & $0.71[0.49,1.03]$ & & & & \\
\hline Hawes 2014 & 0 & 24 & 11 & 37 & $0.7 \%$ & $0.07[0.00,1.07]$ & $\longleftarrow$ & & & \\
\hline Hellstrom 2012 & 594 & 1216 & 1416 & 2758 & $16.9 \%$ & $0.95[0.89,1.02]$ & & & & \\
\hline Walker 2009 & 34 & 358 & 45 & 366 & $11.3 \%$ & $0.77[0.51,1.18]$ & & & & \\
\hline Total $(95 \% \mathrm{Cl})$ & & 5837 & & 13161 & $100.0 \%$ & $0.72[0.57,0.92]$ & & & & \\
\hline \multirow{2}{*}{\multicolumn{7}{|c|}{$\begin{array}{l}\text { Heterogeneity: } \text { Tau }^{2}=0.09 ; \mathrm{Chi}^{2}=42.26, \mathrm{df}=8(\mathrm{P}<0.00001) ; \mathrm{I}^{2}=81 \% \\
\text { Test for overall effect: } Z=2.63(\mathrm{P}=0.009)\end{array}$}} & & & & \\
\hline & & & & & & & 0.01 & $\begin{array}{c}0.1 \\
\text { Favours intervention }\end{array}$ & $\begin{array}{c}10 \\
\text { Favours usual care }\end{array}$ & 100 \\
\hline
\end{tabular}

C Composite rate of readmissions and/or ED visits

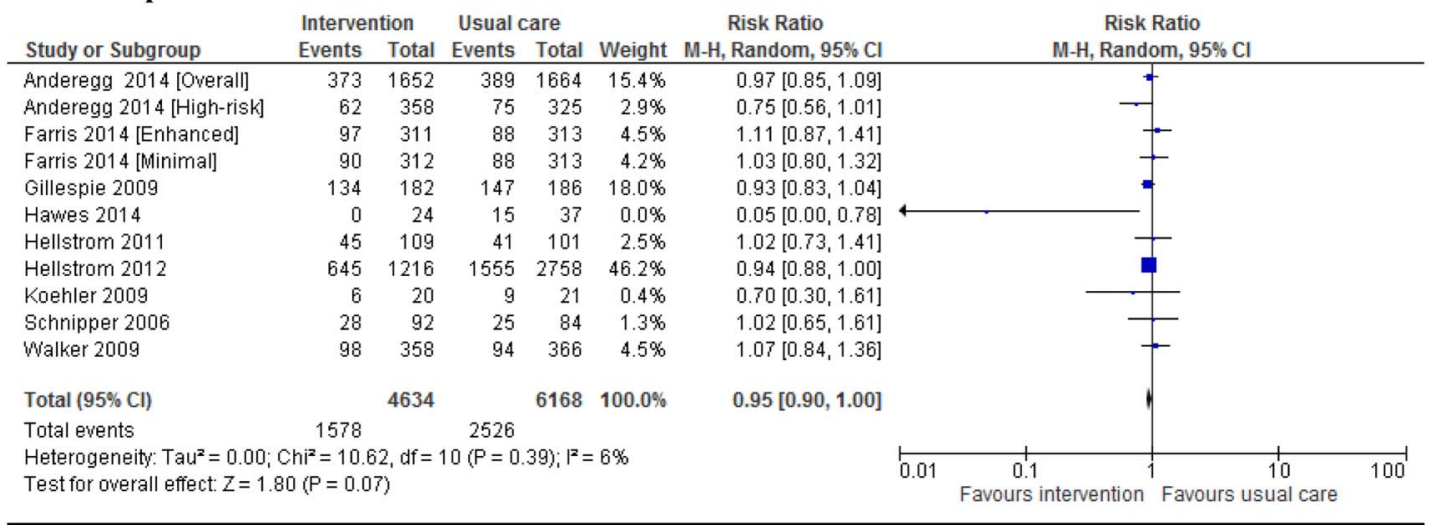

Figure 2 Forest plots of intervention effects on the proportion of patients with all-cause readmission (A), emergency department (ED) visits (B), composite rate of readmissions and/or ED visits (C), adverse drug event-related hospital revisits (D) and mortality (E). Pooled estimates (diamond) calculated by the Mantel-Haenszel random effects model. Horizontal bars and diamond widths represent $95 \%$ Cls. Anderegg et $a^{\beta 5}$ stratified patients into two groups: general population and high-risk patients. Farris et a ${ }^{\beta 7}$ randomised the population into different levels of intervention: minimal and enhanced.

Nine studies $35373940424346-48$ that reported composite all-cause readmission and/or ED visit showed no difference in pooled analysis (RR $0.95 ; 95 \%$ CI 0.90 to 1.00 figure 2C). Only three studies ${ }^{3842} 47$ were meta-analysed for ADE-related hospital revisits. One study ${ }^{46} \mathrm{did}$ not give data in a suitable form. The pooled result showed a substantial reduction of $67 \%$ in hospital revisits (pooled RR $0.33 ; 95 \%$ CI 0.20 to 0.53 ) when pharmacist-led medication reconciliation programmes were implemented (figure 2D). Seven studies ${ }^{37}$ 46-51 gave eight separate sets of data for all-cause mortality that had been reported after 30 days to 12 months of follow-up. However, information on mortality from Bolas et a $\tilde{l}^{0}$ and Farris et $a l^{37}$ was not their primary outcome of interest; nevertheless, we included it in our metaanalysis. Overall, there was no significance difference 
D Adverse drug event-related hospital revisits

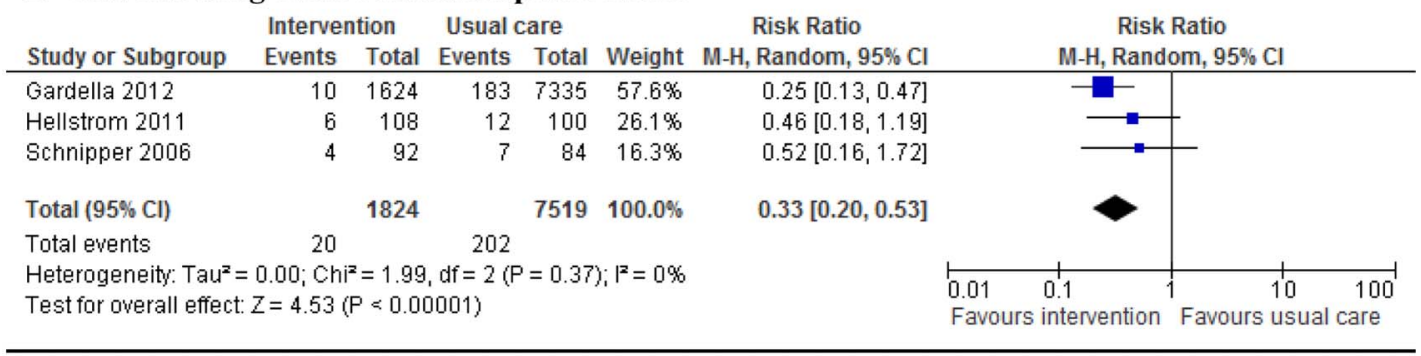

\section{E All-cause mortality}

\begin{tabular}{|c|c|c|c|c|c|c|c|c|c|c|}
\hline \multirow[b]{2}{*}{ Study or Subgroup } & \multicolumn{2}{|c|}{ Intervention } & \multicolumn{2}{|c|}{ Usual care } & \multirow{2}{*}{ Weight } & \multirow{2}{*}{$\begin{array}{c}\text { Risk Ratio } \\
\text { M-H, Random, } 95 \% \mathrm{Cl}\end{array}$} & \multirow{2}{*}{\multicolumn{3}{|c|}{$\begin{array}{c}\text { Risk Ratio } \\
\mathrm{M}-\mathrm{H}, \text { Random, } 95 \% \mathrm{Cl}\end{array}$}} & \\
\hline & Events & Total & Events & Total & & & & & & \\
\hline Bolas 2004 & 17 & 119 & 12 & 124 & $2.0 \%$ & $1.48[0.74,2.96]$ & & & 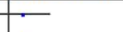 & \\
\hline Farris 2014 [Enhanced] & 12 & 311 & 7 & 313 & $1.1 \%$ & $1.73[0.69,4.32]$ & & & & \\
\hline Farris 2014 [Minimal] & 5 & 312 & 7 & 313 & $0.7 \%$ & $0.72[0.23,2.23]$ & & & - & \\
\hline Gillespie 2009 & 57 & 182 & 61 & 186 & $10.8 \%$ & $0.95[0.71,1.29]$ & & & - & \\
\hline Hellstrom 2011 & 9 & 109 & 9 & 101 & $1.2 \%$ & $0.93[0.38,2.24]$ & & & & \\
\hline Hellstrom 2012 & 330 & 1325 & 685 & 2965 & $73.0 \%$ & $1.08[0.96,1.21]$ & & & & \\
\hline Scullin 2007 & 67 & 371 & 76 & 391 & $10.9 \%$ & $0.93[0.69,1.25]$ & & & - & \\
\hline Stowasser 2002 & 2 & 113 & 3 & 127 & $0.3 \%$ & $0.75[0.13,4.40]$ & & & & \\
\hline Total $(95 \% \mathrm{Cl})$ & & 2842 & & 4520 & $100.0 \%$ & $1.05[0.95,1.16]$ & & & 1 & \\
\hline Total events & 499 & & 860 & & & & & & & \\
\hline $\begin{array}{l}\text { Heterogeneity: } \operatorname{Tau}^{2}=0.0 \\
\text { Test for overall effect: } Z=\end{array}$ & $\begin{array}{l}1 \mathrm{Chi}^{2}=3 \\
1.03(\mathrm{P}=\end{array}$ & $\begin{array}{l}94, \mathrm{df}= \\
30)\end{array}$ & $7(P=0$ & $79) ; 1^{2}=$ & $=0 \%$ & & 0.001 & $\begin{array}{l}0.1 \\
\text { intervention }\end{array}$ & $1 \frac{10}{10}$ & $\overrightarrow{1000}$ \\
\hline
\end{tabular}

Figure 2 Continued.

between the two groups in terms of all-cause mortality (RR 1.05 ; $95 \%$ CI 0.95 to 1.16 ) (figure $2 \mathrm{E}$ ).

\section{Other outcomes}

Studies reporting other clinically important outcomes are summarised in table 2. Some studies ${ }^{46-49}$ furnished information on the proportion of patients who did not revisit the hospital. The intervention group in the three studies ${ }^{4648} 49$ showed a trend towards an increase in the number of patients who did not revisit the hospital for any causes, and the overall pooled analysis was statistically significant (RR 1.10; 95\% CI 1.03 to 1.17 ). There were no significance differences between the intervention and usual care in terms of other relevant clinical outcomes: length of stay after readmission, readmission per patient, ED visit per patient and proportion of patients with ADEs.

\section{Sensitivity analysis}

A one-on-one removal of studies in the meta-analysis did not affect findings in all outcomes except for composite readmission and/or ED visit. A meta-analysis for composite readmission/ED visit showed that only when the study by Faris et al (Enhanced) ${ }^{37}$ or Hawes $e t a l^{39}$ was removed did the result show a significant pooled summary estimate with similar risk ratio (RR 0.95; $\mathrm{p}=0.02$ and 0.03 , respectively).

\section{Subgroup analysis}

Subgroup analysis comparing studies that reported allcause readmissions at earlier versus longer follow-up period showed different patterns of effect: the effect of intervention was not statistically significant for longer follow-up subgroups (RR $0.83,95 \%$ CI 0.68 to 1.06 , $\mathrm{p}=0.14$ ), whereas in earlier follow-up subgroups, the

Table 2 Other clinically relevant outcomes

\begin{tabular}{|c|c|c|c|c|c|c|}
\hline Outcome & $\begin{array}{l}\text { Number of } \\
\text { studies }\end{array}$ & $\begin{array}{l}\text { Number of } \\
\text { patients }\end{array}$ & $\mathbf{R R}$ & Cl & WMD & Cl \\
\hline Patients who did not revisit hospital & 4 & 5314 & $1.10^{*}$ & (1.03 to 1.17$) \dagger$ & & \\
\hline Hospital stay (after readmission) & 2 & 803 & & & -0.57 & $(-5.32$ to 4.17$) \ddagger$ \\
\hline Readmission per patient & 3 & 1370 & & & -0.12 & $(-0.24$ to 0.01$) \ddagger$ \\
\hline ED visit per patient & 2 & 4342 & & & -0.15 & $(-0.53$ to 0.23$) \ddagger$ \\
\hline Patients with ADE & 3 & 1401 & 0.94 & $(0.75$ to 1.20$) \ddagger$ & & \\
\hline
\end{tabular}


effect was significant (RR $0.77,95 \%$ CI 0.60 to 0.98 , $\mathrm{p}=0.03$ ). However, there was no significant difference between these two subgroups. In addition, nonrandomised studies showed a significant reduction in allcause readmission (RR $0.74,95 \%$ CI 0.58 to 0.94 , $\mathrm{p}=0.01$ ) and all-cause ED visit (RR $0.68,95 \%$ CI 0.48 to $0.97, \mathrm{p}=0.03$ ), but there was no difference in terms of study design with these outcomes. As opposed to what has been observed in the entire analysis, the composite outcome seemed to have a slight significant reduction in non-randomised studies (RR $0.95,95 \%$ CI 0.90 to 1.00 , $\mathrm{p}=0.04)$; though there was no difference between the subgroups (see online supplementary appendix D).

\section{Publication bias}

We examined the potential for publication bias by constructing a funnel plot and through statistical tests. There was some indication of asymmetry-particularly for all-cause ED visits-in the funnel plot and, therefore, there was some publication bias, as evidenced by the Egger's $(p=0.04)$ and Begg's tests $(p=0.01)$ in this outcome. We did not find any significant evidence of bias in the other outcomes, as shown by Egger's test value of 0.08 for all-cause readmission, 0.57 for composite readmission/ED visit and 0.83 for all-cause mortality; this was further supported by Begg's test $p$ value of 0.13 , 0.35 and 0.71 , respectively (see online supplementary appendix E).

\section{DISCUSSION}

To the best of our knowledge, this is the first meta-analysis to investigate the effectiveness of pharmacist-led medication reconciliation programmes on clinical outcomes at hospital transitions. This review has shown better outcomes in favour of pharmacist-led interventions. We found a substantial reduction in the rate of all-cause readmissions (19\%), all-cause ED visits (28\%) and ADE-related hospital revisits (67\%). However, pooled data on mortality and composite readmission/ ED visit favoured neither the intervention nor the usual care. Not only were patients allocated to the intervention group readmitted or not only did they revisit the hospital less frequently, but patients free of any events after hospital discharge also increased (RR 1.10; 95\% CI 1.03 to 1.17 ).

No previous reviews have conclusively and consistently shown effectiveness of medication reconciliation interventions, be it in primary care, ${ }^{52}$ long-term settings ${ }^{53}$ or hospital transitions. ${ }^{30} 31$ Particularly, reviews from hospital-initiated medication reconciliation interventions searched the available literature on medication reconciliation strategies and impact on patient safety, and summarised the evidence that medication reconciliation alone was not strong enough to reduce post discharge hospital utilisation. ${ }^{30} 31$ It was not clear to support the effectiveness of such interventions in the hospital setting. However, we believe that the influence of pharmacist's in healthcare utilisation was diluted among those various medication reconciliation strategies and, thus, specifically assessing the effect of pharmacist in medication reconciliation is an important consideration.

Although Thomas et $a b^{54}$ did not find a significant effect in reduction of readmissions due to medicationrelated problems, our review showed that pharmacists' influence in preventing ADE-related hospital revisits was more impactful than any of the outcomes measured. This might be because medication reconciliation picks patients with discontinued medication more powerfully, where this is the case for studies reporting this outcome. ${ }^{4347}$ Other studies also showed that medication discontinuity is the most common reason for discrepancy-related ADE. ${ }^{55}$ Although the study by Gillespie $e t a l^{46}$ was not included in the meta-analysis of this outcome, it showed a much higher reduction of $80 \%$ in medication-related readmissions in the intervention group than in the control group. Readmissions were frequent in earlier follow-up periods. This is as opposed to a review by Kwan et al, ${ }^{31}$ where harm due to medication discrepancies occurred only some months after discharge. However, for most studies, the duration of follow-up was short; only one-third of interventions followed patients for longer than 30 days. Therefore, it might be difficult to come to a conclusion, as there was no sustained benefit from the intervention, and this was supported by non-significant differences between the subgroups. Moreover, non-randomised studies showed a slight significant reduction in all-cause ED visit and readmission and composite outcome, but there was no difference in terms of study design with these outcomes. Otherwise, pooled estimates showed consistent results in all of these three outcomes, regardless of the study design and duration of follow-up. However, care should be taken in interpreting the results as some of the influence of observational studies on the success of outcome was clear, and their heterogeneity should be taken into consideration.

Some of the studies, as part of their intervention, consisted of intermingled components, and the difficulty in ascertaining the success of pharmacist-led intervention is due only to medication reconciliation. After medication reconciliation, for example, medication review as intervention component was added in some studies. Previous systematic reviews that focused on medication review ${ }^{57} 58$ raised a debate as to the impact of medication reviews in general, and pharmacist-led medication reviews in particular. A review by Holland et al, ${ }^{57}$ where only 8 of the 32 included studies were hospital-based and only 2 of these had extensive medical team involvement at hospital transitions, did not support the evidence for pharmacist-led medication review. On the other hand, one of the issues raised in a Cochrane review ${ }^{58}$ was that medication review had varied and wider meaning, and did not stand alone. Prior to medication review, it is medication reconciliation that is practiced routinely at hospital transitions and, thus, considering medication 
review without ensuring the most accurate list of a patient's current medications would be theoretical. This would strengthen our anticipation that interventions with medication reconciliation might be as equally effective as those with mixed interventions.

A number of recent studies have investigated medication reconciliation interventions at the level of real practice models or in integrated management of medicines. ${ }^{4-49}$ Medication reconciliation interventions are complex interventions targeting fragments of services across the entire spectrum of care transitions, and thus take time and effort, but the outcome of safe patient transition is well worth it. This review further consolidates pharmacist-led medication reconciliation programmes might contribute to quality transitions in combinations of those multifaceted components.

\section{Limitation of the study}

There are a number of limitations to this study. First, most studies included high-risk patients, and we did not confirm which patients benefited most from such interventions. Various definitions pertaining to high-risk were employed, including patients with specific disease state, polypharmacy, older age and patients at risk of hospitalisation. Second, interventions target different transitions; we could not take into account this effect in our meta-analysis. For instance, previous prospective studies showed varied results on the rate of medication discrepancies from $30-55 \%$ during admission, ${ }^{59-62}$ to $35-71 \%$ during discharge. ${ }^{46364}$ Coleman et al ${ }^{65}$ showed that patients with medication discrepancies have significantly high rates of readmission. Thus, if this value is extrapolated to clinical outcomes, there might be some variation among studies with respect to these outcomes at the different care transitions. Additionally, few studies were carried out in hospitals where medication reconciliation had already been implemented in some defined areas. Therefore, future studies should evaluate specific areas suited to pharmacist services that would benefit patients the most. Third, most of the studies were single centre evaluations, and there were a few studies with a small number of patients. Considering the success rates within small single centre studies raises an issue about bias. Our included studies were not free of bias and most possessed moderate quality, which leaves the findings open to criticism-for example, Gardella $e t a l^{38}$ in the ADE-related hospital visit, and Hellström et $a l^{48}$ in the mortality forest plots, accounted for a large proportion of the studied subjects, yet these studies possessed low quality score. Fourth, the lack of homogeneity in the data from this meta-analysis confirms the complexity of medication reconciliation and warrants further investigation. We attempted to investigate the sources of variation between studies, but were unable to explain much of it. We were also unable to assess interactions between medication reconciliation and components of interventions. For example, integrated care models may be particularly effective for improving care for some of the interventions, but not for other types, and a pooled analysis would not identify such interactions. Despite these limitations, our meta-analyses showed that interventions that contain one or more elements of medication reconciliation can improve outcomes at hospital transitions.

We also note that only published studies were included in our work. However, the funnel plot asymmetry and statistical tests suggest that the impact of bias was less likely to have a significant effect on the findings. Only articles published in English were assessed for this review. Potentially, there may have been studies, such as that by Sánchez Ulayar et $a l^{66}{ }^{6}$ published in non-English journals, involving interventions for improving care transitions. In addition, research disseminated through the grey literature, such as conference papers and unpublished reports, was not considered.

\section{CONCLUSION}

The results of this meta-analysis indicate that a pharmacist-led medication reconciliation programme at hospital transitions decreases ADE-related hospital revisits, all-cause readmissions and ED visits. However, the effect on mortality and composite all-cause readmission/ ED visit is inconclusive based on the current body of evidence, though improvements in the majority of studies were demonstrated. Future research is needed to assess whether improvements in such outcomes can be achieved with this programme and to determine what/ which components of the intervention are necessary to improve clinical outcomes. Although our results showed that pharmacist-led medication reconciliation was beneficial at care transitions, we still need further research with robust, large randomised control trials of excellent quality to conform our conclusion. Overall, our findings support the implementation of a pharmacist-led medication reconciliation programme that includes some components aimed at improving medication safety.

\section{Author affiliations}

${ }^{1}$ Faculty of Pharmacy, University of Sydney, Sydney, New South Wales, Australia

${ }^{2}$ School of Pharmacy, University of Gondar, Gondar, Ethiopia

${ }^{3}$ Centre for Education and Research on Ageing, Concord Hospital, Sydney, Australia

${ }^{4}$ Faculty of Medicine, St Vincent's Hospital Clinical School, University of New South Wales, Sydney, Australia

Acknowledgements The authors would like to acknowledge Asres Berhan for his comments on the data analysis and interpretation, and statistical advice on using the meta-analysis software. The authors would also like to acknowledge Lorraine Evison for her invaluable contributions in the electronic database searching and abstract screening.

Contributors $A B M$ was responsible for the study conception and design, under the supervision of JEB. The literature search, abstract screening, study and data extraction were undertaken by $A B M$ with further confirmation by JEB. ABM carried out the initial analysis and drafted the first manuscript. JEB and ABM critically reviewed and revised the manuscript. All the authors have read and approved the final manuscript as submitted.

Funding This research received no specific grant from any funding agency in the public, commercial or not-for-profit sectors. 
Competing interests None declared

Ethics approval Our study is an investigation of the literature, and does not need ethical approval for retrieving the already available public content.

Provenance and peer review Not commissioned; externally peer reviewed.

Data sharing statement No additional data are available.

Open Access This is an Open Access article distributed in accordance with the Creative Commons Attribution Non Commercial (CC BY-NC 4.0) license, which permits others to distribute, remix, adapt, build upon this work noncommercially, and license their derivative works on different terms, provided the original work is properly cited and the use is non-commercial. See: http:// creativecommons.org/licenses/by-nc/4.0/

\section{REFERENCES}

1. Institute for Healthcare Improvement. Medication reconciliation review. http://www.ihi.org/resources/Pages/Tools/ MedicationReconciliationReview.aspx (accessed 30 Dec 2014).

2. Rozich JD, Howard RJ, Justeson JM, et al. Standardization as a mechanism to improve safety in health care. Jt Comm J Qual Saf 2004;30:5-14.

3. Cornish PL, Knowles SR, Marchesano R, et al. Unintended medication discrepancies at the time of hospital admission. Arch Intern Med 2005;165:424-9.

4. Wong JD, Bajcar JM, Wong GG, et al. Medication reconciliation at hospital discharge: evaluating discrepancies. Ann Pharmacother 2008:42:1373-9.

5. Pippins JR, Gandhi TK, Hamann C, et al. Classifying and predicting errors of inpatient medication reconciliation. $J$ Gen Intern Med 2008:23:1414-22.

6. Herrero-Herrero JI, García-Aparicio J. Medication discrepancies at discharge from an internal medicine service. Eur J Intern Med 2011;22:43-8.

7. Geurts MM, Talsma J, Brouwers JR, et al. Medication review and reconciliation with cooperation between pharmacist and general practitioner and the benefit for the patient: a systematic review. Br J Clin Pharmacol 2012;74:16-33.

8. Allende Bandrés MÁ, Arenere Mendoza M, Gutiérrez Nicolás F, et al. Pharmacist-led medication reconciliation to reduce discrepancies in transitions of care in Spain. Int J Clin Pharm 2013;35:1083-90.

9. Howard RL, Avery AJ, Howard PD, et al. Investigation into the reasons for preventable drug related admissions to a medical admissions unit: observational study. Qual Saf Health Care 2003;12:280-5

10. Witherington EM, Pirzada OM, Avery AJ. Communication gaps and readmissions to hospital for patients aged 75 years and older: observational study. Qual Saf Health Care 2008;17:71-5.

11. Dedhia $\mathrm{P}$, Kravet $\mathrm{S}$, Bulger J, et al. A quality improvement intervention to facilitate the transition of older adults from three hospitals back to their homes. J Am Geriatr Soc 2009;57:1540-6.

12. Schnipper JL, Hamann C, Ndumele CD, et al. Effect of an electronic medication reconciliation application and process redesign on potential adverse drug events: a cluster-randomized trial. Arch Intern Med 2009;169:771-80.

13. Jack BW, Chetty VK, Anthony D, et al. A reengineered hospital discharge program to decrease rehospitalization: a randomized trial. Ann Intern Med 2009;150:178-87.

14. Joint Commission on Accreditation for Healthcare Organizations. National Patient Safety Goals. 2006. http://www.jointcommission.org/ Improving_Americas_Hospitals_The_Joint_Commissions_Annual Report_on_Quality_and_Safety_-_2006/

15. National Institute for Health and Care Excellence. Technical patient safety solutions for medicines reconciliation on admission of adults to hospital. London, 2007. (NICE/NSPA/2007/PSG001). http://www. nice.org.uk/PSG001 (accessed 30 Dec2014).

16. Canadian Council on Health Services Accreditation. Patient Safety Goals and Required Organizational Practices. Ottawa, 2004. http:// www.accreditation.ca (accessed 30 Dec 2014).

17. Australian Commission on Safety and Quality in Healthcare. Medication reconciliation. http://www.safetyandquality.gov.au/ our-work/medication-safety/medication-reconciliation/ (accessed 30 Dec 2014).

18. Durán-García E, Fernandez-Llamazares CM, Calleja-Hernández MA. Medication reconciliation: passing phase or real need? Inter $J$ Clin Pharm 2012;34:797-802.
19. Canadian Agency for Drugs and Techonlogies in Health. Medication reconciliation at discharge: a review of the clinical evidence and guidelines. 2012. https://www.cadth.ca/medication-reconciliationdischarge-review-clinical-evidence-and-guidelines (accessed $24 \mathrm{Nov}$ 2015).

20. Giménez Manzorro Á, Zoni AC, Rodríguez Rieiro C, et al Developing a programme for medication reconciliation at the time of admission into hospital. Int J Clin Pharm 2011;33:603-9.

21. Schnipper JL, Liang CL, Hamann C, et al. Development of a tool within the electronic medical record to facilitate medication reconciliation after hospital discharge. J Am Med Inform Assoc 2011;18:309-13.

22. Moore $\mathrm{P}$, Armitage $\mathrm{G}$, Wright $\mathrm{J}$, et al. Medicines reconciliation using a shared electronic health care record. J Patient Saf 2011;7:148-54.

23. Bédard $P$, Tardif $L$, Ferland $A$, et al. A medication reconciliation form and its impact on the medical record in a paediatric hospital. $J$ Eval Clin Pract 2011;17:222-7.

24. De Winter S, Vanbrabant $\mathrm{P}$, Spriet I, et al. A simple tool to improve medication reconciliation at the emergency department. Eur $J$ Intern Med 2011;22:382-5.

25. De Winter S, Spriet I, Indevuyst C, et al. Pharmacist- versus physician-acquired medication history: a prospective study at the emergency department. Qual Saf Health Care 2010;19:371-5.

26. Feldman LS, Costa LL, Feroli ER, et al. Nurse-pharmacist collaboration on medication reconciliation prevents potential harm. $J$ Hosp Med 2012;7:396-401.

27. Greenwald JL, Halasyamani L, Greene J, et al. Making inpatient medication reconciliation patient centered, clinically relevant and implementable: a consensus statement on key principles and necessary first steps. J Hospital Med 2010;5:477-85.

28. Eggink RN, Lenderink AW, Widdershoven JW, et al. The effect of a clinical pharmacist discharge service on medication discrepancies in patients with heart failure. Pharm World Sci 2010;32:759-66.

29. Galvin M, Jago-Byrne MC, Fitzsimons M, et al. Clinical pharmacist's contribution to medication reconciliation on admission to hospital in Ireland. Int J Clin Pharm 2013;35:14-21.

30. Mueller SK, Sponsler KC, Kripalani S, et al. Hospital-based medication reconciliation practices: a systematic review. Arch Intern Med 2012;172:1057-69.

31. Kwan JL, Lo L, Sampson M, et al. Medication reconciliation during transitions of care as a patient safety strategy: a systematic review. Ann Intern Med 2013;158:397-403.

32. David Moher D, Liberati A, Tetzlaff J, et al., The PRISMA Group. Preferred Reporting Items for Systematic Reviews and MetaAnalyses: The PRISMA Statement. PLoS Med 2009;6:e1000097.

33. Effective Practice and Organisation of Care (EPOC). [Data collection checklist and risk of bias]. EPOC Resources for review authors. Oslo: Norwegian Knowledge Centre for the Health Services, 2014. http://epoc.cochrane.org/epoc-specific-resources-review-authors (accessed 30 Dec 2014)

34. Higgins JPT, Green S (eds). Cochrane handbook for systematic reviews of interventions. Version 5.1.0 [updated March 2011]. The Cochrane Collaboration, 2011. http://www.cochrane-handbook.org.

35. Anderegg SV, Wilkinson ST, Couldry RJ, et al. Effects of a hospitalwide pharmacy practice model change on readmission and return to emergency department rates. Am J Health Syst Pharm 2014;71:1469-79.

36. Eisenhower C. Impact of pharmacist-conducted medication reconciliation at discharge on readmissions of elderly patients with COPD. Ann Pharmacother 2014:48:203-8.

37. Farris $\mathrm{KB}$, Carter $\mathrm{BL}, \mathrm{Xu} \mathrm{Y}$, et al. Effect of a care transition intervention by pharmacists: an RCT. BMC Health Serv Res 2014:14:406-0.

38. Gardella JE, Cardwell TB, Nnadi M. Improving medication safety with accurate preadmission medication lists and postdischarge education. Jt Comm J Qual Patient Saf 2012;38:452-8.

39. Hawes EM, Maxwell WD, White SF, et al. Impact of an outpatient pharmacist intervention on medication discrepancies and health care resource utilization in posthospitalization care transitions. J Prim Care Community Health 2014;5:14-18.

40. Koehler BE, Richter KM, Youngblood L, et al. Reduction of 30-day postdischarge hospital readmission or emergency department (ED) visit rates in high-risk elderly medical patients through delivery of a targeted care bundle. J Hosp Med 2009;4:211-18.

41. Pal A, Babbott S, Wilkinson ST. Can the targeted use of a discharge pharmacist significantly decrease 30 -day readmissions? Hosp Pharm 2013;48:380-8.

42. Schnipper JL, Kirwin JL, Cotugno MC, et al. Role of pharmacist counseling in preventing adverse drug events after hospitalization. Arch Intern Med 2006;166:565-71. 
43. Walker PC, Bernstein SJ, Jones JN, et al. Impact of a pharmacist-facilitated hospital discharge program: a quasi-experimental study. Arch Intern Med 2009;169:2003-10.

44. Warden BA, Freels JP, Furuno JP, et al. Pharmacy-managed program for providing education and discharge instructions for patients with heart failure. Am J Health Syst Pharm 2014;71:134-9.

45. Wilkinson ST, Pal A, Couldry RJ. Impacting readmission rates and patient satisfaction: results of a discharge pharmacist pilot program. Hosp Pharm 2011;46:876-83.

46. Gillespie U, Alassaad A, Henrohn D, et al. A comprehensive pharmacist intervention to reduce morbidity in patients 80 years or older: a randomized controlled trial. Arch Intern Med 2009;169:894-900.

47. Hellström LM, Bondesson A, Höglund $P$, et al. Impact of the Lund Integrated Medicines Management (LIMM) model on medication appropriateness and drug-related hospital revisits. Eur J Clin Pharmacol 2011;67:741-52.

48. Hellström LM, Höglund $P$, Bondesson $A$, et al. Clinical implementation of systematic medication reconciliation and review as part of the Lund Integrated Medicines Management model-impact on all-cause emergency department revisits. J Clin Pharm Ther 2012;37:686-92.

49. Scullin C, Scott MG, Hogg A, et al. An innovative approach to integrated medicines management. J Eval Clin Pract 2007;13:781-8.

50. Bolas H, Brookes K, Scott M, et al. Evaluation of a hospital-based community liaison pharmacy service in Northern Ireland. Pharm World Sci 2004;26:114-20.

51. Stowasser DA, Collins DM, Stowasser M. A randomised controlled trial of medication liaison services-patient outcomes. J Pharm Pract Res 2002;32:133-40.

52. Bayoumi I, Howard M, Holbrook AM, et al. Interventions to improve medication reconciliation in primary care. Ann Pharmacother 2009;43:1667-75.

53. Chhabra PT, Rattinger GB, Dutcher SK, et al. Medication reconciliation during the transition to and from long-term care settings: a systematic review. Res Social Adm Pharm 2012;8:60-75.

54. Thomas R, Huntley AL, Mann M, et al. Pharmacist-led interventions to reduce unplanned admissions for older people: a systematic review and meta-analysis of randomised controlled trials. Age Ageing 2014;43:174-87.
55. Boockvar KS, Carlson LaCorte H, Giambanco V, et al. Medication reconciliation for reducing drug-discrepancy adverse events. Am J Geriatr Pharmacother 2006;4:236-43.

56. Mergenhagen KA, Blum SS, Kugler A, et al. Pharmacist versus physician-initiated admission medication reconciliation: impact on adverse drug events. Am J Geriatr Pharmacother 2012;10: 242-50.

57. Holland R, Desborough J, Goodyer L, et al. Does pharmacist-led medication review help to reduce hospital admissions and deaths in older people? A systematic review and meta-analysis. $\mathrm{Br} J$ Clin Pharmacol 2008;65:303-16.

58. Christensen M, Lundh A. Medication review in hospitalised patients to reduce morbidity and mortality. Cochrane Database of Syst Rev 2013;2:CD008986.

59. Coffey M, Mack L, Streitenberger K, et al. Prevalence and clinica significance of medication discrepancies at pediatric hospital admission. Acad Pediatr 2009;9:360-5.

60. Gleason KM, Groszek JM, Sullivan C, et al. Reconciliation of discrepancies in medication histories and admission orders of newly hospitalized patients. Am J Health Syst Pharm 2004;61:1689-95.

61. Salanitro AH, Osborn $\mathrm{CY}$, Schnipper JL, et al. Effect of patient- and medication-related factors on inpatient medication reconciliation errors. J Gen Intern Med 2012;27:924-32.

62. Villanyi $D$, Fok M, Wong RY. Medication reconciliation: identifying medication discrepancies in acutely ill hospitalized older adults. Am J Geriatr Pharmacother 2011;9:339-44.

63. Manias E, Gerdtz MF, Weiland TJ, et al. Medication use across transition points from the emergency department: Identifying factors associated with medication discrepancies. Ann Pharmacother 2009;43:1755-64.

64. Grimes T, Delaney T, Duggan C, et al. Survey of medication documentation at hospital discharge: implications for patient safety and continuity of care. Ir J Med Sci 2008;177:93-7.

65. Coleman EA, Smith JD, Raha D, et al. Posthospital medication discrepancies: prevalence and contributing factors. Arch Intern Med 2005;165:1842-7

66. Sánchez Ulayar A, Gallardo López S, Pons Llobet N, et al. Pharmaceutical intervention upon hospital discharge to strengthen understanding and adherence to pharmacological treatment. Farm Hosp 2012;36:118-23. 